\title{
Utilidad de las técnicas nucleares en nutrición: evaluación de la masa grasa corporal y de la ingesta de leche materna
}

\author{
Nuclear techniques in nutrition: assessment of body fat and \\ intake of human milk in breast-fed infants
}

Dra. Anabel Pallaro y Dr. Gabriel Tarduccib

\section{RESUMEN}

Las técnicas nucleares aplicables al área de nutrición son inocuas dado que utilizan isótopos estables. En particular, la dilución isotópica con deuterio evalúa la composición corporal y la ingesta de leche materna. Es un método de referencia para conocer grasa corporal y validar herramientas de bajo costo debido a su exactitud, facilidad de aplicación y por la base de antecedentes de su utilización en adultos y niños. Es no invasiva ya que utiliza saliva como muestra de análisis, lo que la hace aplicable a poblaciones pediátricas. Modificaciones en la grasa se asocian a enfermedades no transmisibles y existen individuos con peso normal pero mayor depósito de grasa. Por ello, es de utilidad analizar sus cambios como instrumento de evaluación en clínica y en programas de salud. Además, esta técnica es la forma exacta de determinar si los lactantes son amamantados exclusivamente mediante seno materno, establecer lactancia materna exclusiva y validar métodos convencionales basados en encuestas a las madres.

Palabras clave: dilución isotópica con deuterio, grasa corporal, ingesta de leche materna.

a. Cátedra de Nutrición. Facultad de Farmacia y Bioquímica.

Universidad de Buenos Aires.

b. Área de Actividad Física, PROPIA, Facultad de Humanidades y Ciencias de la Educación, Universidad Nacional de La Plata.

Correspondencia: Dra. Anabel Pallaro: apallaro@ffyb.uba.ar

Financiamiento: Véase la página 540

Conflicto de intereses: Ninguno que declarar.

Recibido: 17-2-2014 Aceptado: 23-6-2014

\section{ABSTRACT}

The application of nuclear techniques in the area of nutrition is safe because they use stable isotopes. The deuterium dilution method is used in body composition and human milk intake analysis. It is a reference method for body fat and validates inexpensive tools because of its accuracy, simplicity of application in individuals and population and the background of its usefulness in adults and children as an evaluation tool in clinical and health programs. It is a non-invasive technique as it uses saliva, which facilitates the assessment in pediatric populations. Changes in body fat are associated with non-communicable diseases; moreover, normal weight individuals with high fat deposition were reported. Furthermore, this technique is the only accurate way to determine whether infants are exclusively breast-fed and validate conventional methods based on surveys to mothers.

Key words: deuterium dilution technique, body fat, human milk intake.

http:/ /dx.doi.org/10.5546/aap.2014.537

\section{INTRODUCCIÓN}

La estrategia mundial sobre régimen alimentario, actividad física y salud de la Organización Mundial de la Salud (OMS) señala que la carga de enfermedades no transmisibles (ENT) afecta a la población adulta e infantil. ${ }^{1}$ Asimismo, sostiene que la salud y la nutrición de las madres antes y durante el embarazo, la lactancia materna exclusiva (LME) y la alimentación del niño los dos primeros años son importantes para la prevención de enfermedades crónicas a futuro y promueven generaciones de adultos sanos. ${ }^{2}$ Los patrones de crecimiento en la vida temprana prenatal y posnatal se han asociado con sobrepeso, modificaciones de la composición corporal y riesgo de síndrome metabólico en el adulto. ${ }^{3-5}$

Los recientes avances en la capacidad de evaluar los componentes corporales y la ingesta de leche materna ofrecen una oportunidad para mejorar la comprensión de la programación nutricional de la composición corporal y su posible contribución al riesgo de enfermedad posterior. En este sentido, las técnicas nucleares brindan una nueva contribución al campo del conocimiento.

\section{Uso de técnicas nucleares en nutrición}

Las técnicas nucleares son inocuas dado que utilizan isótopos estables. Estos son átomos del mismo elemento químico con igual número atómico o de protones, pero diferente número másico, que es el número de neutrones más protones, y no son radiactivos. 
La radiactividad ocurre en los núcleos inestables que decaen en núcleos más estables y emiten partículas o radiaciones electromagnéticas. Los isótopos estables no emiten radiación y están en la naturaleza en una abundancia variable. Los mayoritarios y más estudiados son los del hidrógeno, carbono, nitrógeno y oxígeno, así como isótopos del hierro, zinc y selenio. ${ }^{6}$

Entre estas técnicas, cabe mencionar la del agua doblemente marcada con deuterio y oxígeno 18, que, mediante recolección de muestras de orina, es utilizada para conocer exactamente el gasto energético y validar metodologías como monitoreo de la frecuencia cardíaca y que fue aplicada por la Organización de las Naciones Unidas para la Agricultura y la Alimentación (FAO, por sus siglas en inglés)/Organización Mundial de la Salud para establecer ingestas recomendadas de energía. ${ }^{7}$ Otras técnicas isotópicas permiten valorar la composición corporal, la ingesta de leche materna, la biodisponibilidad de minerales y las reservas de vitamina A. ${ }^{8,9}$ En particular, la dilución isotópica con deuterio es un método de referencia para conocer grasa corporal y validar herramientas de bajo costo debido a su exactitud, inocuidad y facilidad de aplicación, ${ }^{10}$ además de la amplia base de antecedentes de su utilización en investigación en adultos y niños tanto clínica como de poblaciones.

\section{Uso de la dilución isotópica con deuterio en la evaluación de la grasa corporal}

La evaluación de la composición corporal ha cobrado importancia dado que cambios físicos significativos externos e internos ocurren durante la infancia y la adolescencia. En la clínica, es común la evaluación antropométrica mediante mediciones estandarizadas de peso, talla, circunferencias y pliegues cutáneos y su transformación en índices e indicadores, los cuales tienen correlato con el crecimiento normal y permiten inferir desviaciones. También son usados en encuestas de nutrición para establecer prevalencias de bajo peso, sobrepeso y obesidad. Si bien es aceptada y reconocida esta correlación, los índices antropométricos son relaciones de tamaño y no de composición corporal. Las ENT habitualmente se asocian a modificaciones de la composición corporal y, en general, la masa grasa se encuentra aumentada en individuos con sobrepeso; sin embargo, un incremento puede observarse en individuos con índice de masa corporal (IMC) normal. ${ }^{11}$ La masa grasa es activa y se relaciona con la inflamación, la función vascular, el sistema reproductor y la resistencia a la insulina. ${ }^{12,13}$ Esto sugeriría la necesidad de considerar los cambios de la grasa corporal como herramienta de evaluación en clínica y en programas de salud.

El modelo de dos compartimientos para estimar la composición corporal es el abordaje más sencillo y divide al cuerpo en masa grasa (MG) y masa corporal libre de grasa (MCLG). Los métodos de evaluación se diferencian por su aplicabilidad en pacientes o población, validez, procedimiento, calibración y precisión. Las características propias de la población pediátrica determinan la selección de los métodos. La exposición a la radiación debe evitarse, así como la toma de muestras de sangre cuando sea posible. De manera sencilla, se pueden estimar los compartimientos corporales por ecuaciones de predicción mediante, por ejemplo, los pliegues cutáneos o determinaciones de impedancia eléctrica, siempre que estén validadas para la población en estudio.

El deuterio es un isótopo estable del hidrógeno presente en el cuerpo, los alimentos y el agua en $0,015 \%$, y un adulto tiene aproximadamente $6 \mathrm{~g}$ de deuterio. En bioquímica, se lo utiliza como trazador en moléculas para estudiar reacciones químicas, cambios metabólicos y componentes corporales, ya que químicamente se comporta como el hidrógeno, del que se distingue por su masa por espectrometría de masa o infrarroja. ${ }^{6} \mathrm{Al}$ administrar este isótopo, se produce enriquecimiento de los fluidos, hecho que se utiliza para su identificación.

FIgURA 1. Dilución con deuterio ${ }^{14}$

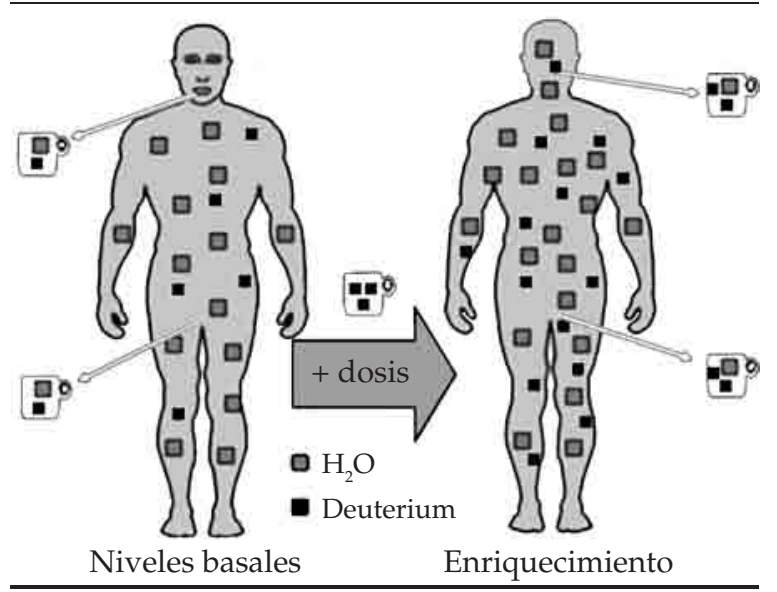


La técnica consiste en suministrar agua deuterada oralmente, la que se mezcla con el agua corporal, se equilibra en horas y se metaboliza por la misma vía que el agua y se elimina por orina, saliva, sudor y leche materna. ${ }^{14}$ (Figura 1). El enriquecimiento y determinación de deuterio en saliva permite la estimación del agua corporal total (ACT) para luego calcular la MCLG mediante el coeficiente de hidratación y finalmente el porcentaje de MG. ${ }^{15}$ Es una técnica sencilla y no invasiva, ya que permite utilizar saliva como muestra de análisis.

El agua es el componente corporal más abundante y varía entre $80 \%$ al nacer y $45 \%$ en el obeso. ${ }^{16}$ Es el medio necesario para las reacciones bioquímicas y el transporte de nutrientes y hormonas. Pequeñas modificaciones en el ACT pueden producir un cambio mensurable en el peso corporal, de manera que el ACT se convierte en un parámetro central y sensible en la evaluación de la composición corporal. A diferencia de otros componentes a nivel molecular, el compartimiento acuoso se constituye de una sola especie química, lo que simplifica la medición por el principio de dilución.

La administración de deuterio es inocua para los seres humanos. La cantidad administrada en estudios de composición corporal e ingesta de leche humana enriquece el agua corporal con un máximo de $0,1 \%$ en la madre y menos de la mitad en el bebé. En este nivel, no se han reportado efectos secundarios adversos, los que comenzarían a observarse en concentraciones excesivamente altas en tejidos, mayores de $15 \% .15,17$

En el marco de proyectos latinoamericanos de las Naciones Unidas/Organismo Internacional de Energía Atómica (OIEA), hemos desarrollado la técnica en el país, y estudios preliminares de tipo descriptivo-transversal en niños argentinos de ambos sexos entre 6 y 12 años de edad ( $n=$ 250) han demostrado elevados porcentajes de sobrepeso, obesidad y MG, concomitantemente con cierto grado de inflamación y modificación del perfil lipídico, condiciones asociadas al síndrome metabólico. Si se considera el sobrepeso/ obesidad como una acumulación excesiva de grasa perjudicial para la salud, aproximadamente $8-10 \%$ de este grupo se estaría subestimando al utilizar solamente el IMC como indicador..$^{18-20}$

\section{Uso de la dilución isotópica con deuterio en la evaluación de la ingesta de leche materna}

A partir de datos retrospectivos del momento de introducción de alimentos, se puede estimar el porcentaje de lactantes que, habiendo iniciado la lactancia materna, no recibieron ningún alimento hasta el sexto mes de vida. Esto permite inferir el porcentaje que pudo ser potencialmente alimentado con leche materna. ${ }^{21}$ Se ha reportado que los métodos convencionales basados en encuestas a las madres podrían estar sujetos a sesgos de memoria. ${ }^{22}$ La dilución isotópica con deuterio permite evaluar con exactitud la ingesta de leche materna y, por lo tanto, la LME o predominante y permitiría validar cuestionarios utilizados para evaluar las prácticas de alimentación infantil. ${ }^{23,24}$

El volumen de leche materna consumida por el lactante se evalúa durante un período de 14 días utilizando el método de "dosis a la madre", que administra agua deuterada a la madre y sigue la desaparición de su compartimento acuoso y la aparición en el del lactante; para ello, se recogen muestras de saliva de ambos. La técnica, descrita por Andy Coward en 1982, también permite la estimación del consumo de agua de otras fuentes y la determinación de la composición corporal materna. ${ }^{15,25}$ Esta metodología está siendo ensayada por nuestro grupo; no es engorrosa, pero requiere la participación activa de los pares mamá-bebé y del personal involucrado, ya que la toma de saliva del bebé puede llevar más tiempo que con los niños y adultos.

\section{Utilidad de la dilución isotópica con deuterio en programas de nutrición y salud}

En consonancia con la transición epidemiológica, varios países latinoamericanos registran prevalencias crecientes de obesidad. En Argentina, según la Encuesta Nacional de Nutrición y Salud (ENNyS), la prevalencia de sobrepeso y obesidad es de $31,5 \%$ y $10,4 \%$ en niños de 6 meses a 5 años y de 24,9\% y 19,4\% en mujeres de 19 a 49 años. ${ }^{26}$ Según la Encuesta de Factores de Riesgo, la actividad física baja aumentó de $46,2 \%$ a $54,9 \%$; la obesidad, de $14,6 \%$ a $18,2 \%$; y la diabetes, de $8,40 \%$ a $9,60 \%$ desde 2005 a $2009,{ }^{27}$ lo que evidencia la carga de malnutrición relacionada con el exceso de peso y las ENT. Además, la ENNyS mostró que el 95,4\% de los bebés recibía leche materna al momento del alta de la maternidad. De acuerdo con el último estudio de Situación de Lactancia Materna en Argentina de 2011, el 60\% de los bebés de 2 meses reciben lactancia materna exclusiva, proporción que se reduce al $45 \%$ al $4^{\circ}$ mes y al $30 \%$ a los 6 meses de edad. ${ }^{21}$ Adicionalmente, se observó que más del 70\% de los niños de 6 a 23 
meses había consumido, al menos, $20 \%$ o más de su requerimiento energético y que un $45 \%$ de ellos había superado el 50\% de sus necesidades. ${ }^{28}$ En este contexto, existiría la posibilidad de cambios en la composición corporal de los niños y predisposición a desarrollar en el futuro alteraciones metabólicas asociadas a las ENT. El abandono temprano de la LM adquiere relevancia en la instauración de la intolerancia a la glucosa, el síndrome metabólico y la diabetes tipo 2. Las mediciones precisas de la ingesta diaria de leche materna serían útiles para la identificación de dichas asociaciones y la conformación de las intervenciones dirigidas a satisfacer el crecimiento sin generar acumulación de grasa corporal y modificaciones metabólicas relacionadas con las causas más frecuentes de enfermedad.

Resumiendo, la utilización de estas técnicas permite la evaluación del depósito de grasa en niños y adultos, así como contribuye al conocimiento de la ingesta de leche materna y su posible influencia en la composición corporal tanto del niño como de la madre. A nivel epidemiológico, contribuye con la validación de herramientas sencillas, como ecuaciones de predicción de masa grasa locales y de cuestionarios de lactancia, ambos de potencial aplicabilidad en programas de nutrición. Ecuaciones preliminares de masa grasa validadas con la dilución isotópica con deuterio han sido obtenidas por nuestro grupo mediante mediciones antropométricas sencillas y de bajo costo y mediciones de impedancia eléctrica. ${ }^{29,30}$ Actualmente, se dispone de un protocolo estandarizado que maximiza la exactitud y precisión de medición, a disposición del equipo médico como de programas.

La aplicación regional de estas técnicas ha contribuido a programas nacionales; en Chile, se observó que la tasa de obesidad en niños preescolares asistentes a guarderías se redujo de $10,4 \%$ a $8,4 \%$ entre 2000 y 2010 a través de un programa de alimentación y actividad física de la Junta Nacional de Auxilio Escolar y Becas y de Jardines de Infantes, cuya fase piloto fue evaluada con el soporte de la técnica de dilución isotópica por el Instituto de Nutrición y Tecnología de los Alimentos (INTA)/Universidad de Chile, adoptado por las autoridades nacionales y extendido entre 2006 y 2010 al 75\% de los preescolares. ${ }^{9}$ También se han generado ecuaciones nacionales de predicción de composición corporal basadas en el método de dilución isotópica en México. ${ }^{31}$ Además, la mayoría de los países en el mundo están experimentando una reducción alarmante en la frecuencia y consistencia de la LME, y se ha reportado que el uso de isótopos estables detectó que solo $13 \%$ de los niños fue alimentado mediante pecho durante los primeros 6 meses en lugar del $27 \%$ según la evaluación por cuestionario. ${ }^{4}$

De la experiencia adquirida, debe destacarse la importancia de generar un ambiente de confianza entre el paciente, sus padres y el equipo de salud interdisciplinario involucrado, explicando claramente qué es el agua deuterada, la información adicional que se obtiene con el estudio y la ausencia de riesgos para la salud. En todos los estudios, se dispuso de un protocolo aprobado por Comité de Ética, asentimiento del niño y consentimiento informado firmado por los padres y se mantuvieron talleres informativos con docentes, padres y niños. El trabajo en terreno, almacenamiento, transporte de las muestras y determinación analítica se desarrollaron exitosamente, por lo que la aplicación de la metodología por primera vez en el país resultó altamente satisfactoria y abre perspectivas de transferencia.

\section{Financiamiento}

Los autores agradecen la financiación de la capacitación del grupo de investigación al Organismo Internacional de Energía Atómica (OIEA, Proyectos RLA 6064, RLA 6071 y RLA 6059), Universidad de Buenos Aires (UBACYT, B098 y 20020100100255) y Universidad Nacional de La Plata, así como la contribución económica para la adquisición del equipamiento para la medición analítica por la cátedra de Nutrición a la Facultad de Farmacia y Bioquímica de la UBA y el OIEA.

\section{REFERENCIAS}

1. Organización Mundial de la Salud. Estrategia mundial sobre régimen alimentario, actividad física y salud. Ginebra: OMS; 2004.

2. World Health Organization. Global strategy for infant and young child feeding: the optimal duration of exclusive breastfeeding. Fifty-Fourth World Health Assembly. Geneva; 2001. Disponible en: http://apps.who.int/gb/ archive/pdf_files/WHA54/ea54id4.pdf. [Acceso: 23 de junio de 2014].

3. Wells JC, Chomtho S, Fewtrell MS. Programming of body composition by early growth and nutrition. Proc Nutr Soc 2007;66(3):423-34.

4. Medoua GN, Sajo Nana EC, Ndzana AC, Makamto CS, et al. Breastfeeding practices of Cameroonian mothers determined by dietary recall since birth and the dose-tothe-mother deuterium-oxide turnover technique. Matern Child Nutr 2012;8(3):330-9. 
5. Gale C, Logan KM, Santhakumaran S, Parkinson JR, et al. Effect of breastfeeding compared with formula feeding on infant body composition: a systematic review and metaanalysis. Am J Clin Nutr 2012;95(3):656-69.

6. Wolfe R. Basic characteristics of isotopic tracers. En: Radioactive and stable isotope tracers in biomedicine: principles and practice of kinetic analysis. New York: Wiley-Liss; 1992. Págs.1-18.

7. World Health Organization, Food and Agriculture Organization of the United Nations, United Nations University. Human energy requirements. Report of a Joint FAO/WHO/UNU Expert Consultation. Roma, 1724 October 2001. Rome: FAO, 2004.

8. Davidsson L, Tanumihardjo S. New frontiers in science and technology: nuclear techniques in nutrition. Am JClin Nutr 2011;94(2):691S-5S

9. International Atomic Energy Agency. IAEA Bulletin. Contributing solutions for Nutrition. Viena, 2014:2024. Disponible en: http://www.iaea.org/Publications/ Magazines/Bulletin/Bull551/index.html. [Acceso: 23 de junio de 2014].

10. Lukaski HC, Johnson PE. A simple, inexpensive method of determining total body water using a tracer dose of $\mathrm{D}_{2} \mathrm{O}$ and infrared absorption of biological fluids. Am JClin Nutr 1985;41(2):363-70.

11. Okorodudu DO, Jumean MF, Montori VM, RomeroCorral A, et al. Diagnostic performance of body mass index to identify obesity as defined by body adiposity: a systematic review and meta-analysis. Int J Obes (Lond) 2010;34(5):791-9.

12. Bray GA. Medical consequences of obesity. JClin Endocrinol Metab 2004;89(6):2583-9.

13. Madeira FB,Silva AA, Veloso HF, Goldani MZ, etal. Normal weight obesity is associated with metabolic syndrome and insulin resistance in young adults from a middle-income country. PloS One 2013;8(3):e60673.

14. International Atomic Energy Agency. Assessment of body composition and total energy expenditure in humans using stable isotope techniques. IAEA Human Health Series $\mathrm{N}^{\circ}$ 3. Viena; 2009.

15. International Atomic Energy Agency. Introduction to body composition assessment using the deuterium dilution technique with analysis of saliva samples by Fourier Transform Infrared Spectrometry. IAEA Human Health Series 12. Viena; 2011.

16. Schoeller DA. Hydrometry. En: Heymsfield SB, Lohman TG, Wang Z, Going SB, eds. Human Body Composition. $2^{\text {nd }}$ ed. Champaign, Illinois: Human Kinetics; 2005.Págs.35-49.

17. Jones PJ, LeatherdaleST. Stable isotopes in clinical research: safety reaffirmed. Clin Sci (Lond) 1991;80(4):277-80.

18. Slater C, Mokhtar N, Valencia M, Ruiz V, etal. Contribution of nuclear techniques to nutrition programmes in Latin America [abstract]. En: 20 ${ }^{\text {th }}$ International Congress of Nutrition; 2013, Septiembre 15-20, Granada, España. Ann Nutr Metab 2013;63(Suppl 1):304.

19. Fernández I, Vidueiros SM, Nápoli C, Paganini A, et al. Body fat mass determination of argentinean children by deuterium dilution technique and its relationship to lipid profile [abstract]. En: $20^{\text {th }}$ International Congress of Nutrition; 2013, Septiembre 15-20, Granada, España. Ann Nutr Metab 2013;63(Suppl 1):1196.

20. Nápoli C, Vidueiros S, Paganini A, Dimarco G, et al. La doble carga de la malnutrición en un grupo de escolares argentinos de 6 a 11 años [abstract]. En: LVIII Reunión Científica Anual dela Sociedad Argentina de Investigación Clínica;2013,23-23 denoviembre; Mar del Plata, Argentina. Medicina (B Aires) 2013;73(Supl III):108.

21. Argentina. Ministerio de Salud, Dirección Nacional de Maternidad e Infancia. Situación de la lactancia materna en Argentina. Año 2011. Buenos Aires, 2011. Disponible en: http://www.msal.gov.ar/images/stories/bes/ graficos/0000000215cnt-a11a-Situacion-de-la-lactanciamaterna-2011.pdf . [Acceso: 23 de junio de 2014].

22. Piwoz EG, Creed de Kanashiro H, Lopez de Romaña G, Black RE, et al. Potential for misclassification of infants' usual feeding practices using 24-hour dietary assessment methods. J Nutr 1995;125(1):57-65.

23. Cisse SA, Bluck L, Diaham B, Dossou N, et al. Use of Fourier transformed infrared spectrophotometer (FTIR) for determination of breastmilk output by the deuterium dilution method among Senegalese women. Food Nutr Bull 2002;23(3 Suppl):138-41.

24. Haisma H, Coward WA, Albernaz E, Visser GH, et al. Breast milk and energy intake in exclusively, predominantly, and partially breast-fed infants. Eur J Clin Nutr 2003;57(12):163342.

25. Coward WA, Cole TJ, Sawyer MB, Prentice AM. Breast-milk intake measurement in mixed-fed infants by administration of deuterium oxide to their mothers. Hum Nutr Clin Nutr 1982;36(2):141-8.

26. Argentina. Ministerio de Salud. Encuesta Nacional de Nutrición y Salud. Documento de resultados 2007. Buenos Aires, 2007. Disponible en: http://www.msal.gov.ar/ images/stories/bes/graficos/0000000257cnt-a08-ennysdocumento-de-resultados-2007.pdf. [Acceso: 23 de junio de 2014].

27. Argentina. Ministerio deSalud.Segunda Encuesta Nacional deFactores deRiesgo para Enfermedades No Transmisibles. Buenos Aires, 2011. Disponible en: http://www.msal. gov.ar/ent/images/stories/vigilancia/pdf/fr_encuestanacional-factores-riesgo-2011.pdf. [Acceso: 23 de junio de 2014].

28. Argentina. Ministerio de Salud. La alimentación de los niños menores de 2 años. Resultados de la Encuesta Nacional de Nutrición y Salud - ENNyS 2010. Buenos Aires, 2010. Disponible en: http://www.msal.gov.ar/ promin/publicaciones/pdf/la_alimentacion_de_los_ ninos_menores_de_2_anos.pdf. [Acceso: 23 de junio de 2014]

29. Tarducci GO. Estudio de la composición corporal en niños y su relación con la actividad física: desarrollo de ecuaciones de predicción de masa grasa y masa libre de grasa [Tesis Doctoral]. A Coruña, España: Universidade da Coruña, 2012. Disponible en: http://ruc.udc.es/dspace/ bitstream/2183/11543/2/Tarducci_GabrielOmar_ TD_2013.pdf. [Acceso: 12 de mayo de 2014].

30. Vidueiros SM, Tarducci G, Morea G, Bardach A, et al. Assessment of body composition in children by bioelectrical impedance and skinfold anthropometry. FASEB J 2010;24:732.5.

31. Ramírez E, Valencia ME, Bourges H, Espinosa T, etal. Body composition prediction equations based on deuterium oxide dilution method in Mexican children: a national study. Eur J Clin Nutr 2012;66(10):1099-103. 